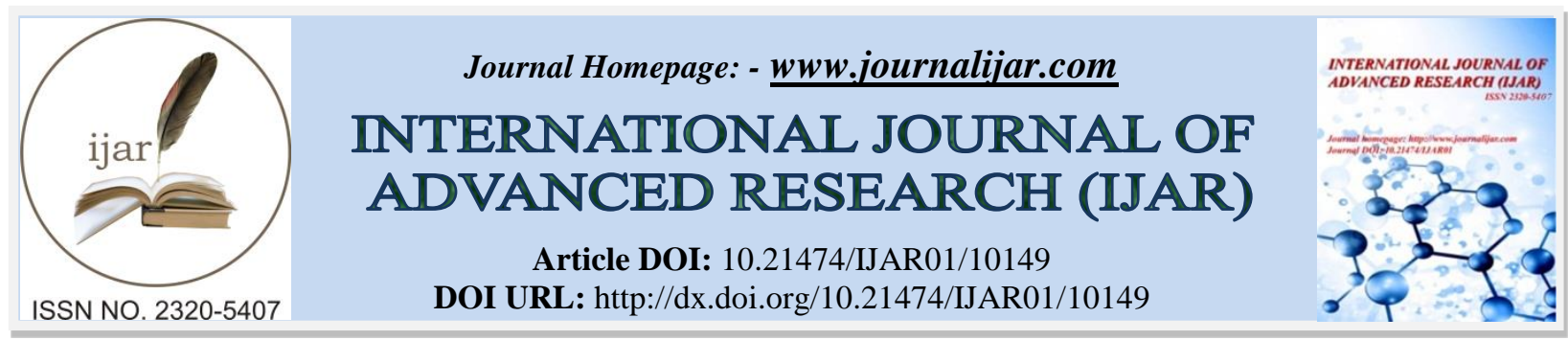

RESEARCH ARTICLE

\title{
DESIGN OF A MONITORING AND CONTROL SYSTEM IN THE BIODIESEL PURIFICATION PROCESS.
}

\author{
Rahmawati $^{1,2}$, Taufik Djatna ${ }^{2}$, Erliza Noor ${ }^{2}$ and Irzaman ${ }^{2}$.
}

1. Department of Electrical Engineering, Lhokseumawe State Polytechnic, Aceh, 24375, Indonesia.

2. Department of Agricultural Industrial Engineering, IPB University, Bogor, West Java, 16002, Indonesia.

\section{Manuscript Info}

Manuscript History

Received: 03 October 2019

Final Accepted: 05 November 2019

Published: December 2019

Key words:-

Biodiesel Purification, Bpmn, Monitor and Control Systems.

\section{Abstract}

Methyl esters in the transesterification process containing impurities can cause problems when they are used and stored. Some things that are strictly related to the biodiesel purification process are the monitoring and control process. A real-time monitoring system with multiple sensors and controls in the purification process is needed to ensure temperature, $\mathrm{pH}$, level and dielectric can be achieved in optimal washing and drying times. This study aims to provide a conceptual model design of monitoring and control systems in the biodiesel purification process through a needs analysis using Business Process Modeling and Notation. The conceptual model in the biodiesel purification process that was built with BPMN produced four stakeholders who integrated each other and outlined each task and rule to optimize each function. The formulation in the monitoring and control system recommends the installation of Arduino and Xbee-based sensors that can be accessed through the Local Area Network. The system has been designed to facilitate the monitoring process in realtime and accurately.

Copy Right, IJAR, 2019,. All rights reserved.

\section{Introduction:-}

The process of biodiesel purification today is a technology that needs to be studied more deeply. Methyl esters in the transesterification process containing impurities can cause problems when they are used and stored. The methyl esters produced from the transesterification reaction must be purified to reduce the concentration of contaminants such as the remaining catalyst, water, and glycerol to an acceptable level. Based on the ASTM D2709 standard the maximum required residual water is $0.05 \%$ vol (EN12938: $500 \mathrm{mg} / \mathrm{kg}$ ), methanol 0.2\% volume and glycerol $0.24 \%$ mass. Some things that are closely related to the biodiesel purification process are the monitoring and control process.

There are several effects of process variables in biodiesel purification, such as temperature and time requirements in the reactor that affect the purification efficiency. The process of washing biodiesel can produce undesirable emulsification due to mixing that is too strong. The residual water content and temperature need to be monitored regularly to improve the efficiency and quality of biodiesel. For the purification process to run optimally, the biodiesel flow rate, washing water flow rate, stirring speed, and temperature must be controlled.

Corresponding Author:- Rahmawati.

Address:- Department of Electrical Engineering, Lhokseumawe State Polytechnic, Aceh, 24375, Indonesia. 
A real-time monitoring and control system using multiple sensors in the purification process is needed to ensure temperature, $\mathrm{pH}$, and water content can be achieved in the washing and drying phase repetition. Some research on controlling the process of making biodiesel has been done by used an adaptive control system in the transesterification process based on temperature sensors using Fuzzy range control (Fonseca et al. 2016). The biodiesel reaction is non-linear and dynamic requires an efficient control algorithm to handle variations in operating process parameters and the effect of interference on the biodiesel reactor system. Fuzzy control is preferred because it is more adaptive to intervention than PID control (Wali et al. 2011). The automatic control system is built based on the transfer function (output and input comparison) of the biodiesel purification process.

In business process management, the interaction between process models and decisions plays an important role because decisions based on running processes will influence the results of processes. The requirement to produce information in real time from a lot of data prompted researchers to create a monitoring and control system for biodiesel purification processes by integrating the configuration of sensors, communication systems, Fuzzy-PID control systems and actuators in the network. Bazhenova et al. (2019) has modeled the data in the BPMN process and used it to make health care process decisions. Research objectives: (1) Provide a conceptual model design of the monitoring and monitoring system of the biodiesel purification process through needs analysis using Business Process Modeling and Notation (BPMN 2.0) and (2). Formulating a monitoring and control system with WSN (wireless sensor network) in the biodiesel purification process.

\section{Literature Review:- \\ System Mechanism:}

The analysis and design mechanism refers to the process of examining business situations to improve through specific procedures and methods. System analysis and design are related to performance improvement and achievement of goals for profitability and growth (Ko et al. 2009). Look at a system and determine how adequate its function is, changes that must be made, and the quality of output is part of the system analysis. With analysis and design, the production system can be increased inefficiency and effectiveness.

A system is presented in the form of a system-building entity consisting of inputs/outputs, processes, rules, opportunities, threats, physical boundaries, resources, stakeholders, controls, roles, missions, and goals. The process in a business system is analogous to a system entity that represents the top-level system, sub-system, and other derivatives (Dijkman et al. 2011). This system entity has limited ability to process inputs into outputs with specific performance measures. During processing, the input into the system output will be influenced by factors from the operating environment such as stakeholders and their respective roles, rules, system mission, opportunities, threats, controls, and resources. The resulting output can be either an appropriate or non-conforming output, which will give reciprocity to the system.

Business Process Modeling Notation 2.0 (BPMN 2.0) is used as a tool to describe the behavior and interactions between actors in a system. Notation from BPMN 2.0 is generally divided into flow objects, Connecting Objects, Swimlanes, and artifacts (White dan Miers 2008). Flow object consists of 3 objects, namely, events, activities, and gateways are the main elements of the business process model in BPMN 2.0. The event is represented as a circle and means something that will "happen." Activities are notations that symbolize a process in the business process model. Activities can be decomposed into more detailed processes into sub-processes. The gateway controls the sequential flow of a process and can combine or separate an input/output flow according to gateway conditions. Put a gateway is conditional in the form of if-else. Flow objects are connected using connecting objects. Swimlanes are a visual mechanism of the organization and categorization of activities, based on cross-functional flow diagrams. Swimlanes consist of two types, pool, and lanes. The pool represents significant participants in a process, explicitly separating different organizations /actors/stakeholders. A pool consists of two or more lanes.

\section{Wireless Sensor Network:}

The development of the Wireless Sensor Network (WSN) system is a network composed of various sensor nodes that have sensing, wireless communication, and computing capabilities. Wireless Sensor Network (WSN) is an embedded system equipment in which there are one or more sensors and was equipped with communication system equipment (transceiver). Autonomous sensors are distributed to monitor physical or environmental conditions such as temperature, sound, and pressure. This sensor is used as a tool to control industrial processes, health monitoring in hospitals, and etc (Anuja dan Murugeswari 2013). WSN network is composed of various sensor nodes that have sensing capabilities, wireless communication, and computing. A sensor node has two components, namely mote, and 
sensor. The sensor is always attached to the object, which is responsible for storage, computing, and communication. The sensor is used to capture or gather the information that is following the characteristics of the sensor. Data that has been collected from several sensor nodes is transmitted via wireless communication to the central base station for further processing.

In the use of a larger scale, Zigbee technology is known as wireless sensor networks and data processing using Arduino microcontrollers. Zigbee technology is a wireless communication technology that has advantages, including low power consumption, high fault tolerance, and flexibility (Yanfei et al. 2009). Arduino Leonardo microcontroller module with XBee socket functions as a data processor that is read by sensors in the form of analog data to then be converted into digital data. Leonardo's microcontroller module with Xbee socket is already equipped with a 10-bit analog to digital converter (ADC) circuit. The output from the sensor can be directly connected to the analog pin on the board, and this module is equipped with an Xbee Socket. With this socket, users can use it together with Xbee, Wifi, Bluetooth, and other RF modules.

The XBee receiver is used to receive data wirelessly from the router sensor node module. The Xbee receiver is connected to the host, the XBee adapter module, with an interface that can be directly related to the XBee receiver pins, thus allowing the XBee receiver to be directly paired. The primary function of the XBee USB adapter module is as a bridge that connects the XBee receiver to the serial port on the computer. The Xbee radio frequency module requires a regulator module because the working voltage of $3.3 \mathrm{~V}$ is different from the $\mathrm{V}_{\mathrm{CC}}$ microcontroller which is $5 \mathrm{~V}$.

\section{Methods:- \\ Conceptual Design Model:}

This study uses a graphic conceptual system design with a standard business process model approach that is Business Process Modeling Notation 2.0 (BPMN 2.0) to describe the behavior and interactions between actors in the process of monitoring and controlling purification biodiesel production. System verification is carried out with the help of Sybase Power Designer 16.5 software to check whether the system model conceptually designed has met the system component requirements to be able to operate according to the system entity proposed by Wasson (2005).

Verification is done by checking the error and warning in the software whereas the validation of the conceptual system designed for this research was carried out by checking the requirements needed to design a monitoring and controlling system for the purification process of biodiesel production so that the system intended had fulfilled the requirements previously designed.

\section{Monitoring and Control System Formulation with WSN}

Information or data was collected, then sent and displayed to the process operator. Data from sensors placed at the plant are fed to the microcontroller and Xbee to be transmitted wirelessly to the PC server functions as a display monitoring and setpoint regulator. The setpoint is transmitted wirelessly to the controller system (microcontroller) for processing data and giving commands to the actuator. Actuators carry out orders to act on biodiesel purification plants. An user interface is provided that allows users to access and manipulate machines to optimize the process.

\section{Result And Discussion:- Needs Analysis:}

Analysis of system requirements is carried out to determine the system design needs of the model that must be prepared for the system to run. The approach used in this study is a system entity based on the concept of the view of a system in the form of interoperability. The inputs, processes, and outputs analyzed in this system design based on the method illustrated in Figure 1.

\section{Conceptual Design Model for Biodiesel Purification with BPMN:}

This stage begins by identifying components to provide a holistic picture of the achievement of objectives. The purification of biodiesel involves a washing and drying process. Stakeholders that can be identified in biodiesel purification are washing reactors, drying reactors, monitoring, and controlling sensors. Each stakeholder has a role that can be optimized by considering rules and controls on the biodiesel purification process parameters. The threat of noisy sensor data and the formation of emulsions in biodiesel must be minimized as little as possible to optimize opportunities for the development of monitoring and control systems. 


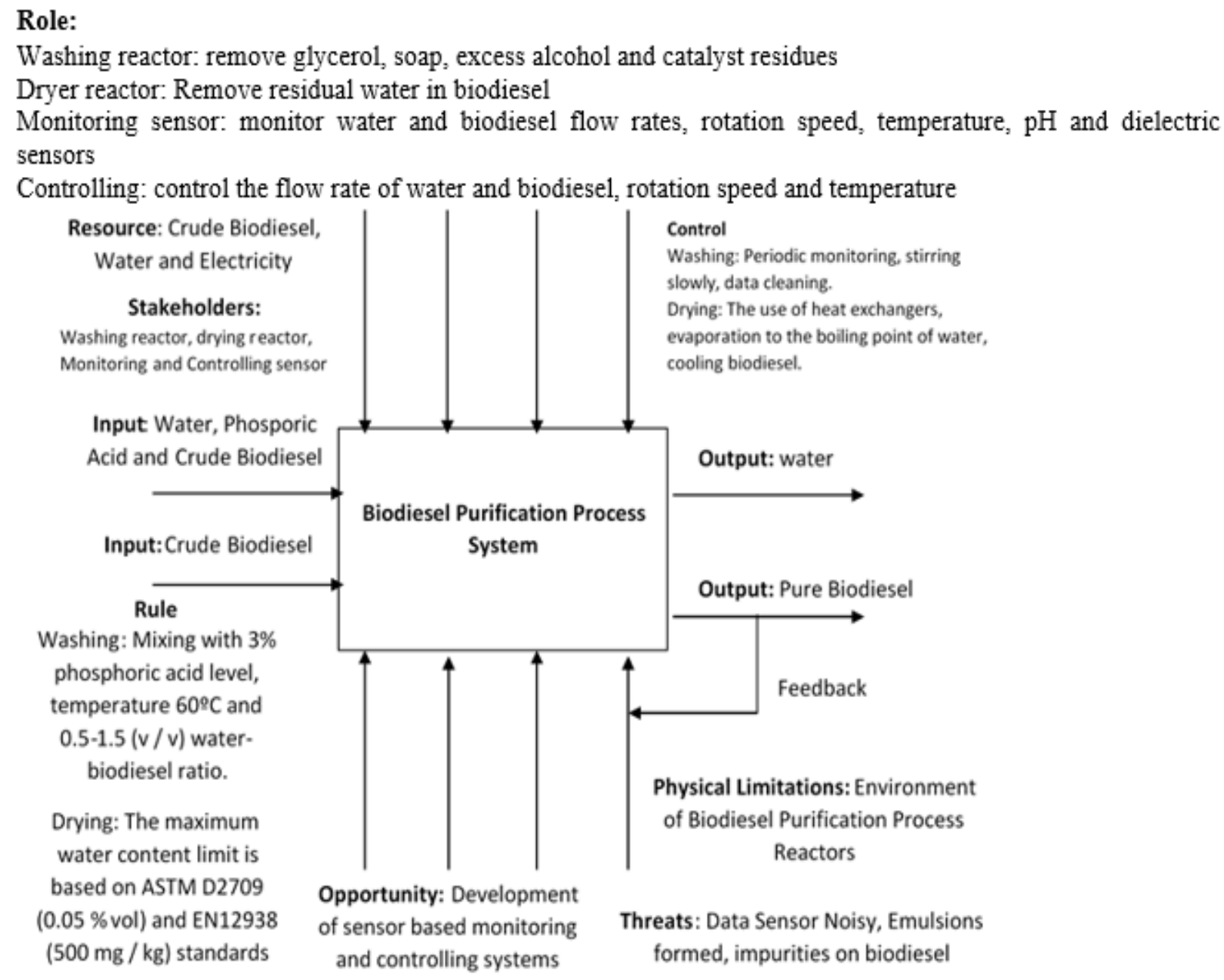

Figure 1:- Biodiesel Purification Process Diagram

In general, wet washing tank reactors use water to separate methanol, glycerol, and catalyst residues because these components are easily dissolved in water. In this process the system is designed to regulate the flow rate of crude biodiesel and water that enters the reactor. Two objects/actors in the monitoring and control system at the reactor involved are control (sensor and inlet volume regulator) and washing.

Monitoring the washing process involves identifying the amount of impurities and the volume of raw biodiesel to determine the amount of washing water needed. It was carried out with hot distilled water at a temperature of $60{ }^{\circ} \mathrm{C}$ with a ratio of water and biodiesel 0.5: 1 . It process was repeated until the wash water reaches $\mathrm{pH}$. Testing to find out the time to stop washing is carried out measurements of the remaining washing water with a neutral $\mathrm{pH}$ indicator that shows the washing water does not contain contaminants from biodiesel. Mehta dan Duran (2015) stated that washing biodiesel with water (wet washing) can remove methanol and glycerol, which qualify the requirements of EN 14214 and ASTM D6751. An important point to note in this case is that the pump flow rate must be low enough to keep the water settled and separate from biodiesel. The mixing technique in biodiesel washing uses a mechanical mixer to speed up water and biodiesel contact; however, high-intensity mixing can result in the formation of unwanted emulsions. Wet washing technique with the addition of water and slow agitation has done to prevent emulsions. Forming an emulsion or soap can cause product loss and is relatively difficult to separate (Knothe 2005). After each aspect was identified, the conceptual model was designed in BPMN to find out the communication flow of each process. The business process model illustrated in Figure 2. 


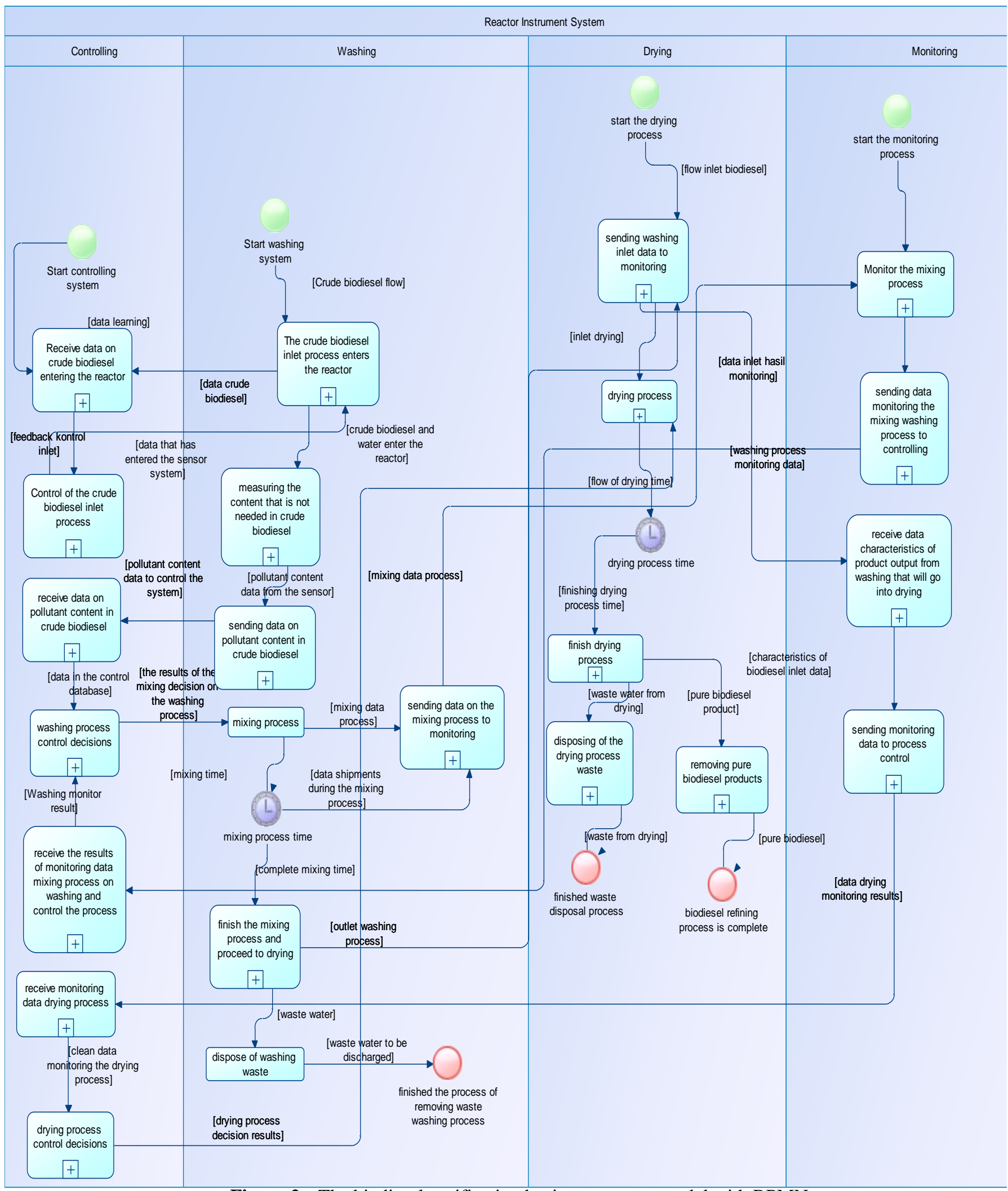

Figure 2:- The biodiesel purification business process model with BPMN 
The monitoring the washing process results are sent to the controller to control the desired process conditions further. In order to avoid emulsions and hydrolysis, it is necessary to use optimal water, temperature, and processing time. Techniques used to measure the condition and quality of biodiesel, such as water content and $\mathrm{pH}$, require relatively complex, slow, and expensive laboratory equipment. Fast and inexpensive testing is needed to be able to carry out biodiesel quality sensing directly when production takes place using sensors and control systems automatically.

After the washing process completed, it is continued at the drying process stage to qualify the requirements for a minimum amount of remaining water in biodiesel. The impurities have a more substantial phase and are at the bottom, while the light phase biodiesel is channeled into the drying reactor. The drying phase is needed to remove water trapped in the biodiesel after the washing process. The remaining biodiesel water causes hydrolysis (FFA formation) and bacterial growth, which can cause machine corrosion, where the maximum water content limit is based on ASTM D2709 (0.05 vol\%) and EN12938 standard (500 mg/kg). The possibility of FFA formation due to hydrolysis of esters due to the presence of water and the establishment of emulsions or soap can cause product loss and are relatively difficult to separate (Knothe 2005). The drying process is carried out by evaporation by heating water containing biodiesel to a water vapor point of $120{ }^{\circ} \mathrm{C}$. The use of heat exchangers aims to facilitate the vacuum to attract water vapor in biodiesel. Besides, the vacuum also serves to reduce levels of methanol and soap in methyl esters. Measurement of biodiesel water content generally uses the Karl Fischer method. After the drying process, biodiesel was cooled as the final product.

Variables that influence the purification process are temperature, time, biodiesel solvent ratio, and the condition of raw biodiesel. Rahayu dan Mindaryani (2007) stated that data information is sent to the actuator to optimize the control system by performing work orders automatically. Indicators of the success of this process are $\mathrm{pH}$, residual water content, and methanol residue. The main criteria in the form of residual water, soap, and catalyst must be analyzed in the final biodiesel product. The $\mathrm{pH}$ parameter is used as an indicator of the residual catalyst during the washing process, while the remaining water is an indicator of the drying process. The control process is carried out as an effort to optimize the use of water and the duration of the biodiesel purification process. Therefore automatic control of the purification process is needed to optimize the process by increasing the efficiency and quality of biodiesel.

BPMN 2.0 verification on the design of this conceptual model is done with the help of Sybase Power Designer 16.5 software. Verification and validation aim to show that a system is well in accordance with its specifications and provides the expected function in accordance with the objectives (Djatna et al. 2018). Verification of the BPMN model that was built shows that there were no errors and no warnings were found.

\section{Closed Loop Purification Control System for Biodiesel:}

In the process of biodiesel purification, there are many obstacles in the production quality targets that are not constant and lack efficiency. The control system is used to optimize system performance in overcoming process interruptions such as equipment performance interruptions. Here are some critical points in the control system. The monitoring results are sent to the controller to control the desired process conditions further. Techniques used to measure the condition and quality of biodiesel, such as water content require relatively complex, slow, and expensive laboratory equipment. Fast and inexpensive testing is needed to be able to carry out biodiesel quality sensing directly when production takes place using sensors and control systems automatically.

In the process of monitoring the process of biodiesel purification requires a capacitance sensor to measure the dielectric as a purity measurement of biodiesel. K-type thermocouple temperature sensor to monitor the temperature of biodiesel as a feedback control heater that provides heat. Dielectric sensors monitoring the process of biodiesel purification use a capacitance effect to recognize a material by assuming the material is dielectric on a capacitor. Glycerol biodiesel contaminants, water, methanol, and soap are all polar while non-polar biodiesel so that water can remove these contaminants from biodiesel. Water is used to wash biodiesel because biodiesel contaminants can dissolve completely in more polar solvents. This is supported by Noor et al. (2015) which states that the solubility of a substance is caused by the polarity of its solvent. Dielectric value is influenced by the polarity of a material.

Figure 3 shows a block diagram during the drying process. The sensor senses the process variable temperature of biodiesel $(\mathrm{T})$ and dielectric biodiesel $(\mathrm{K})$. Temperature and dielectric information from the sensor is processed by the transmitter and sent to the controller in the form of a signal. In controllers, measurable process variables are 
compared with setpoints $\left(\mathrm{R}_{\mathrm{T}}\right.$ and $\left.\mathrm{R}_{\mathrm{K}}\right)$. The difference value based on size, length, and speed of error. Temperature controllers perform calculations according to the control algorithm to produce a control signal in the form of an electrical signal and sent to the final control element. Dielectric measurement has several advantages when compared with other measurement methods. Besides being fast, it also does not damage the nature, is relatively simple, and uses a low power level (Irzaman et al. 2014).

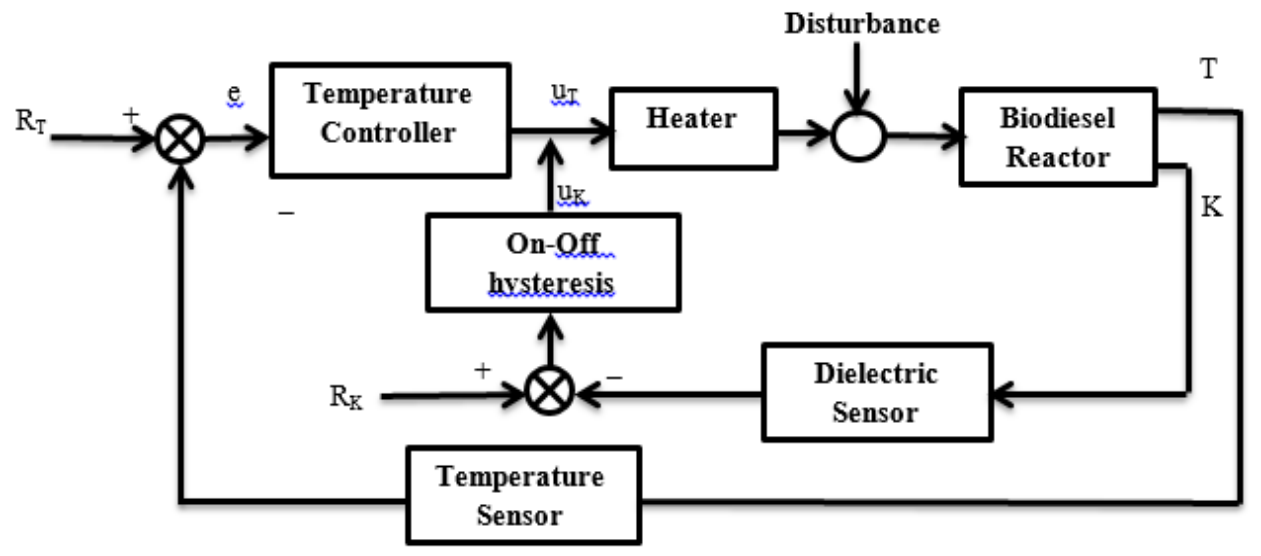

Figure 3:- Sensor and Control System information flow formulation

The control signal $\left(\mathrm{u}_{\mathrm{T}}\right.$ and $\mathrm{u}_{\mathrm{K}}$ ) to process a controlled variable so that it becomes the same as the set point. A change in the control signal causes a change in heater power. This change causes a difference in the heating power variable. In this case, the heater, which has been in a steady-state, is disturbed in the form of an increase in flow rate and biodiesel temperature. This will cause changes in dielectric and temperature output.

Temperature and dielectric sensors measure the process variables, then the data is sent by the transmitter and fed back to the controller. The measured feedback signal is subtracted from the setpoint to produce an error. The controller calculates errors through the controller algorithm to produce a control signal. The control signal acts on Pulse Width Modulation (PWM) to change the manipulated variable. Changes in manipulated variables are used to keep process variables measured at setpoint values from changes in disturbance variables. PWM method for heating regulation by adjusting the percentage of high pulse width to the period of a square signal in the form of a periodic voltage applied to the heater driver as a heater. The greater the ratio of the signal high with the signal period, the greater the heating power given to the heater.

Process control aims to maintain the temperature of biodiesel at the desired value (setpoint). This is done to minimize disturbances in the form of flow rates of biodiesel inlet, biodiesel temperature, and heat loss. Temperature and dielectric biodiesel are referred to as controlled variables. The control mechanism starts with measuring the temperature and dielectric of biodiesel. The measurement results were compared with the desired value (setpoint). Based on the difference, determined what actions will be taken. When the biodiesel temperature is lower than the desired temperature, the heater power is increased. Otherwise, the heater power is reduced. When the biodiesel dielectric is as desired, the heater is turned off.

Simulation of temperature control using Simulink is shown in Figure 4. The biodiesel temperature set point is 110 ${ }^{\circ} \mathrm{C}$. The plant (Gp) used based on Elkhtem et al. (2014) is a crude distillation unit controller.

$\mathrm{Gp}=\frac{-0.3654 \mathrm{~S}+0.657}{\mathrm{~S}^{2}+1.642 \mathrm{~S}+1.982}$

Model of temperature transmitter using thermocouple sensor (Noriyanto dan Musyafa 2019). The controller calculates errors through the controller algorithm to produce a control signal. The control system uses a Fuzzy logic controller by adjusting the heating power based on error and delta error. The MIMO process is difficult to directly design in a fuzzy controller because too many rules will be involved. MIMO fuzzy controller approach is done by decouple MIMO controller approach into several SISO controllers. Fuzzy controller consists of a fuzzy temperature 
controller and fuzzy relative humidity controller on self-tuning fuzzy controller for air-conditioning systems (Xiaoqing 2004).

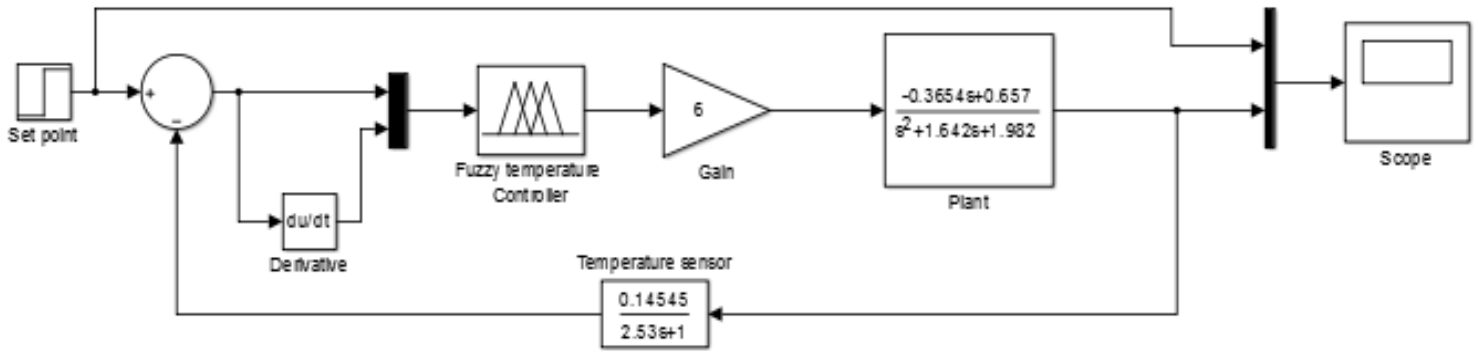

Figure 4:- Simulation of temperature control

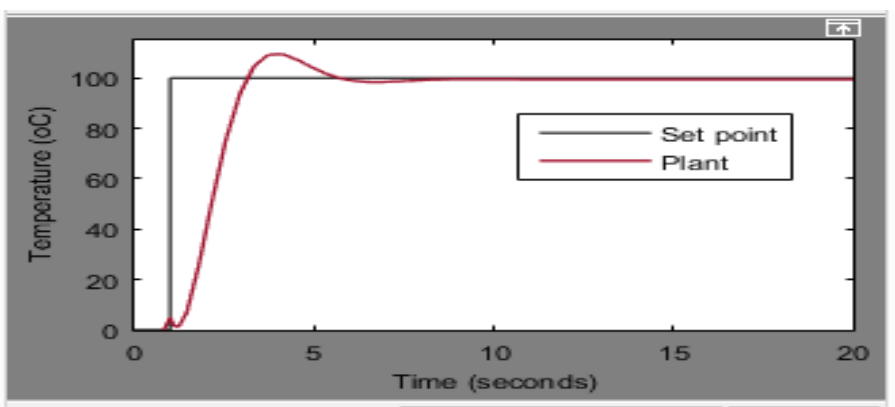

Figure 5:- Time response for Fuzzy Logic controllers

Response performance in Figure 5 shows that it produces 9\% overshoot, because this system works on biodiesel drying so this overshoot does not cause biodiesel to evaporate. Error steady state is $0 \%$, and the rise time is 5 seconds. Thus the fuzzy control applied meets the temperature regulation criteria for drying biodiesel.

\section{Monitoring System Formulation with WSN:}

Alternative solutions are made in the form of instrumentation system and central control system design formulations. The instrumentation system consists of sensor input, local data processing and actuator output. The central control system consists of a wireless communication module and a computer as the central controller. An automation system is one of the methods used to prevent the failure of the final product and improve the quality of biodiesel to be more effective and efficient. The ideal, reliable and inexpensive method of monitoring biodiesel quality is expected to be easier to apply and faster to monitor reactions in real time. More fully explained in Fig 6.

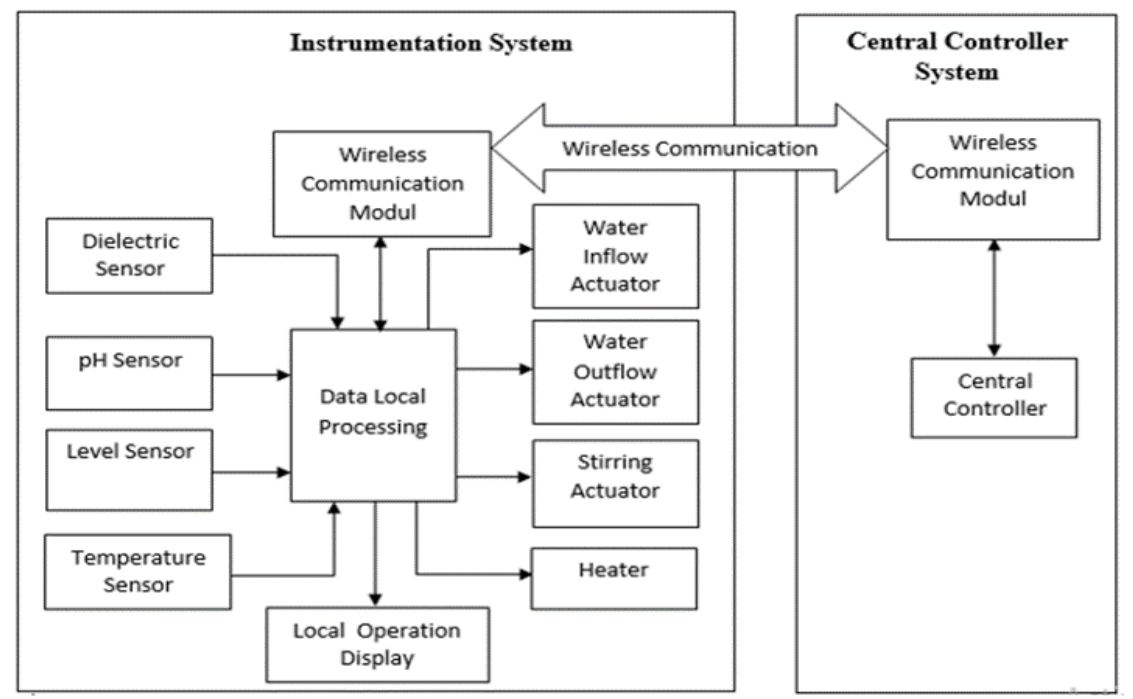

Figure 6:- Formulation Design of a Monitoring System for Biodiesel Purification Process 
Data transfer using wireless communication between two devices and sensor network applications using the Xbee module. Communication settings by creating a source address (MY) and network identity (ID) on the PAN coordinator (Personal Area Network). Creating a source address (MY) on the PAN coordinator aims to ensure the destination address (DL) of all network devices. In contrast, the creation of a unique network identity at the PAN coordinator aims to ensure that other nearby networks do not use the PAN network identity in the radio area, wherein that area radio equipment can communicate with other radios. The next step is making the source address (MY), destination address (DL), and network identity (ID) on all devices. The purpose of making the destination address (DL) on the device is to ensure that data packets from the device can be received by the coordinator, this time the coordinator acts as the data collector of all the devices in the network. Figure 7 represents the framework in the monitoring system.

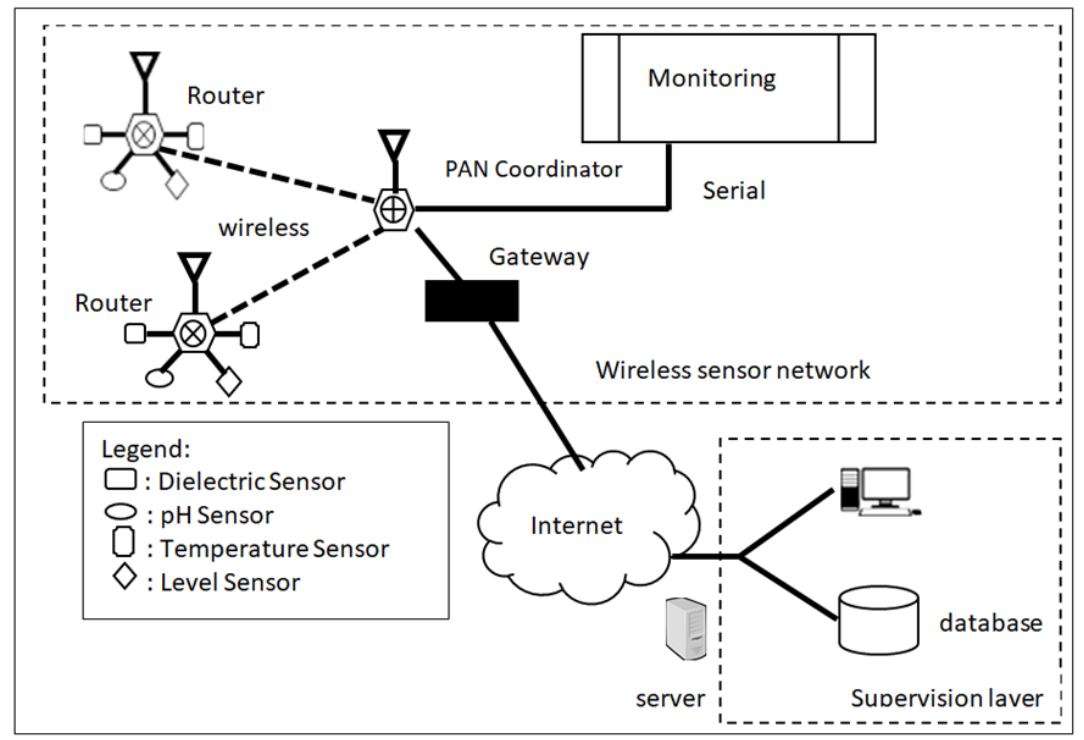

Figure 7:- Monitoring System Framework

Apart from being a transmitter, Xbee transmitters are also used to transmit data wirelessly from the location of the prototype sensor node router to the server computer. The XBee receiver that functions as a receiver has the same specifications as the XBee transmitter in the sensor node of the router. The Xbee receiver is connected to the host, the XBee adapter module, with an interface that can be directly connected to the XBee receiver pins, thus allowing the XBee receiver to be directly paired. The primary function of the XBee USB adapter module is as a bridge that connects the XBee receiver to the serial port on the computer (Central Controller System).

A user interface allows users to access and manipulate machines to optimize process operations. In order to processing data by sensor, it must be programmed in advance of the Arduino microcontroller module. Processing data read by the sensor must first program the Arduino microcontroller module using IDE (Integrated Development Environment) software, in this implementation using the $\mathrm{C}$ programming language and the process of compiling the program using the Arduino IDE.

The stages of wireless data acquisition at the receiving and sending parts are the sensor coordinator and router nodes. A node in the sensor network includes a microcontroller, data storage, sensor, analog-to-digital converter (ADC), a data transceiver, and an energy source. Nodes are connected using architectures called network topologies such as stars, tree clusters, and mesh. In different topologies, sensor nodes can act as data transmitters and receivers or routers that work in multi-hop mode. Wireless Sensor Networks using adaptive data acquisition have good flexibility and scalability, and are able to simultaneously achieve good energy efficiency and high-quality sensing from critical events (Rao et al. 2019). Figure 8 shows information collected then sent and displayed to the process operator.

Data from sensors placed on the plant are fed to the microcontroller and Xbee to be transmitted wirelessly to the PC server. The communication standard used is based on Zigbee. PC server functions as a display monitoring and 
setpoint regulator. The setpoint is transmitted wirelessly to the controller system (microcontroller) for processing data and giving commands to the actuator. Actuators carry out orders to act on biodiesel purification plants. A user interface is provided that allows users to access and manipulate machines to optimize process operations.

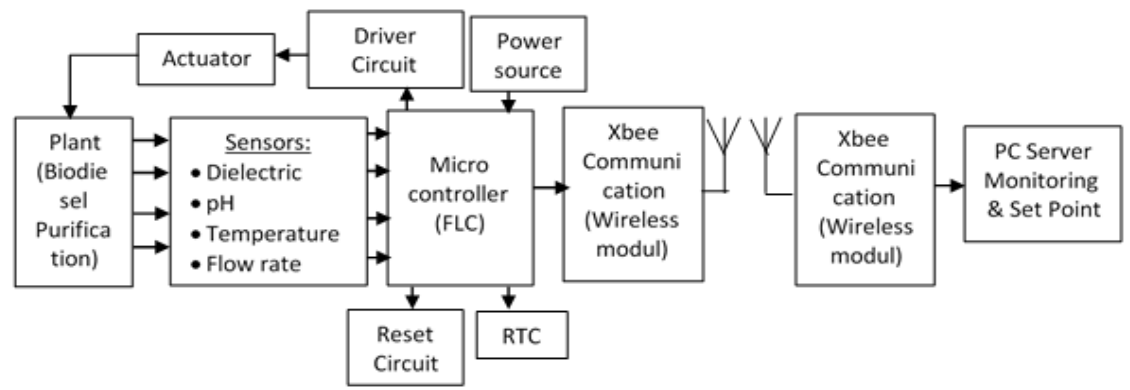

Figure 8:- Sensor and Control System information flow formulation

ZigBee network protocol is used to send data from the node to the gateway. The Zigbee tranciever model is given in Figure 9. The ZigBee network consists of transmitters and receivers. The network has two operations to receive control signals (accept_data) from the gateway and ask the node to send data, and the other operation is to send data using the ZigBee protocol (Gorantla dan Mani 2015). Figure 9 shows a ZigBee transceiver model using simulink.

This block of error rate calculation produces a vector of length three, whose entries correspond to the error rate, the total number of errors (the number of instances that an Rx element does not match the corresponding Tx element), and the total number of comparisons that the block made.

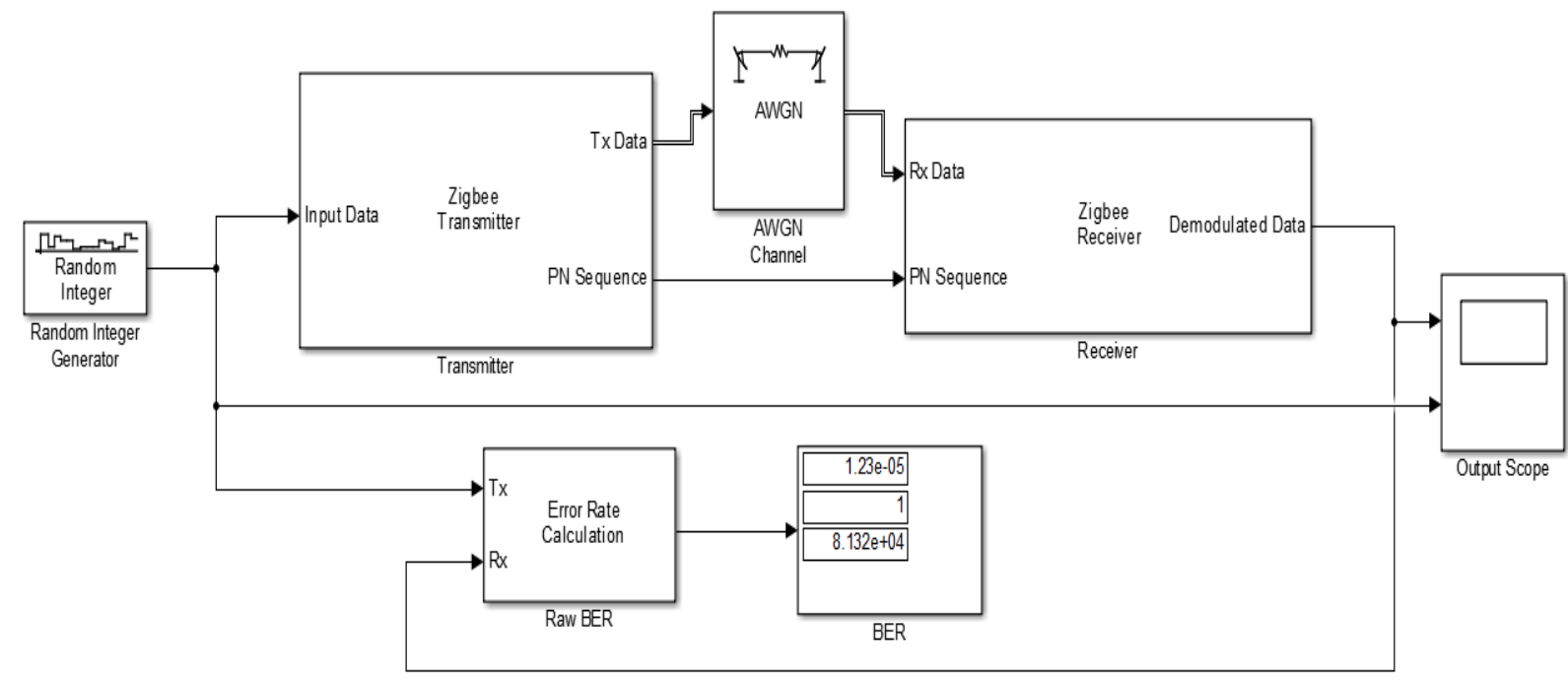

Figure 9:- ZigBee transciever model

The comparison of transmitter and receiver signal waves with AWGN is shown in Figure 10. The bit error rate (BER) is the number of bit errors per unit time. The bit error ratio (BER) is the number of bit errors divided by the total number of transferred bits during a studied time interval. Bit error ratio is a unitless performance measure, often expressed as a percentage. BER values $10^{-6}$ have met the requirements that must be met are BER $10^{-6}-10^{-12}$. 



Figure 10:- Comparison between input and output bits for error bits calculation

\section{Conclusion:-}

The conceptual model in the biodiesel purification process that was built with BPMN produced four stakeholders who were mutually integrated and outlined each task and rule to optimize each function. The monitoring and control system formulation requires Arduino and Xbee-based sensors that can be accessed through the local area network. The system built facilitates the monitoring process in real-time and accurately.

\section{Acknowledgement:-}

This research has been supported by the BPPDN Grant, the Ministry of Research Technology and the Higher Education Republic of Indonesia in Domestic Doctorate Scholarship Program.

\section{References:-}

1. Anuja P, Murugeswari T. 2013. A novel approach towards Building Automation through DALI-WSN Integration. International Journal of Scientific and Research Publications. 3(4)

2. Bazhenova E, Zerbato F, Oliboni B, Weske M. 2019. From BPMN process models to DMN decision models. Information Systems. 83:69-88

3. Dijkman R, Hofstetter J, Koehler J. 2011. Business Process Model and Notation: Springer.

4. Djatna T, Anggraeni E, Sailah I. 2018. An Analysis and Design of Downstreaming Decision System on Palm Oil Agroindustry Based on Multilabel Classification. 2018 International Conference on Advanced Computer Science and Information Systems (ICACSIS), IEEE.

5. Elkhtem SS, Gasmelseed G, Karama B. 2014. Transfer function identification and tuning of crude distillation unit controller at Khartoum refinery-Sudan. Journal of Applied and Industrial Sciences.

6. Fonseca RR, Frattini Fileti AM, Franco IC, da Silva FV. 2016. Experimental fuzzy/split-range control: novel strategy for biodiesel batch reactor temperature control. Chemical Engineering Communications. 203(9):12511259

7. Gorantla K, Mani V. 2015. Simulink model for Zigbee transceiver using OQPSK modulation under fading channels. 2015 International Conference on Communications and Signal Processing (ICCSP), IEEE.

8. Irzaman, Agustina A, Khabibi J. 2014. Electrical properties of Indonesian hardwood case study: Acacia mangium, Swietenia macrophylla and Maesopsis eminii.

9. Knothe G. 2005. Dependence of biodiesel fuel properties on the structure of fatty acid alkyl esters. Fuel Processing Technology. 86(10):1059-1070.doi:10.1016/j.fuproc.2004.11.002.

10. Ko RK, Lee SS, Wah Lee E. 2009. Business process management (BPM) standards: a survey. Business Process Management Journal. 15(5):744-791

11. Mehta A, Duran SK. 2015. Canola biodiesel: an experimental investigation for production of biodiesel and performance measurement in diesel engine. International Journal of Research in Engineering and Technology. $4(4): 535-541$

12. Noor E, Harmi L, Maddu A, Yusron M. 2015. Fabrication of nanogingerol by combining phase inversion composition and temperature. RESEARCH JOURNAL OF PHARMACEUTICAL BIOLOGICAL AND CHEMICAL SCIENCES. 6(1):38-47

13. Noriyanto RD, Musyafa A. 2019. Reliability and safety study on heat exchanger control system: Case study in ortho xylene process-petrochemical industry. AIP Conference Proceedings, AIP Publishing. 
14. Rahayu SS, Mindaryani A. 2007. Optimization of biodiesel washing by water extraction. Proceedings of the world congress on engineering and computer science, WCECS San Francisco, USA.

15. Rao Y, Zhao G, Wang W, Zhang J, Jiang Z, Wang R. 2019. Adaptive Data Acquisition with Energy Efficiency and Critical-Sensing Guarantee for Wireless Sensor Networks. Sensors. 19(12):2654

16. Wali W, Cullen J, Hassan K, Mason A, Al-Shamma'a A. 2011. Comparison between Adaptive and Fuzzy logic controllers for advance microwave biodiesel reactor. 2011 IEEE Symposium on Computers \& Informatics, IEEE.

17. Wasson CS. 2005. System analysis, design, and development: Concepts, principles, and practices: John Wiley \& Sons.

18. White SA, Miers D. 2008. BPMN modeling and reference guide: understanding and using BPMN: Future Strategies Inc.

19. Xiaoqing Z. 2004. Self-tuning fuzzy controller for air-conditioning systems

20. Yanfei L, Cheng W, Chengbo Y, Xiaojun Q. 2009. Research on zigbee wireless sensors network based on modbus protocol. 2009 International Forum on Information Technology and Applications, IEEE. 